\title{
Retinal Ganglion Cell Axons Recognize Specific Guidance Cues Present in the Deafferented Adult Rat Superior Colliculus
}

\author{
Mathias Bähr and Andrea Wizenmann \\ Neurologische Universitätsklinik and Max-Planck-Institut für Entwicklungsbiologie, D-72076 Tübingen, Germany
}

During development, retinal ganglion cell axons establish a topographically ordered projection from the retina to the superior colliculus (SC). The putative guidance activities for retinal axons that operate during embryonic development are not detectable in the normal adult SC. However, these cues reappear upon transection of the optic nerve of adult rats. In the present study, we used a modified version of the "stripe assay," in which membranes from either anterior or posterior SC alternated with laminin stripes. Temporal embryonic retinal axons consistently avoid membranes from embryonic posterior SC, but only rarely from adult deafferented SC. However, they are attracted to membranes from both embryonic and adult deafferented anterior SC. Nasal retinal axons only show a significant preference for membranes from posterior SC after deafferentation. When retinal axons were offered a choice to grow on membranes either from their embryonic or their deafferented

The retinotectal projection has been widely used as a model to examine the mechanisms that underlie the formation of precise topographic patterns. During development of the rat visual system, a topographic retinotectal projection is formed: retinal ganglion cell (RGC) axons from the temporal retina project to the anterior (rostral) superior colliculus (SC), whereas nasal retinal axons project to the posterior (caudal) SC. Ventrally and dorsally located RGCs establish a similar projection along the mediolateral axis of the SC. To analyze the role of putative molecules involved in axonal guidance and target recognition, Walter et al. (1987a) developed an in vitro assay (stripe assay). In this assay, retinal axons were grown on carpets, which consist of alternating stripes of membranes derived from anterior and posterior embryonic tectum (Walter et al., 1987a,b).

In all tested species (chick, mouse, fish, and rat), temporal retinal axons avoid growing on membrane stripes from the posterior SC, whereas nasal retinal axons did not show a growth preference (Walter et al., 1987a,b; Godement and Bonhoeffer, 1989; Vielmetter and Stuermer, 1989; Roskies and O'Leary, 1994). Using this assay, two different membrane-bound putative guiding molecules were identified in the chick, both of which are likely to be involved in steering retinal axons on the chick tectum

\footnotetext{
Received Jan. 24, 1996; revised May 20, 1996; accepted May 28, 1996.

This work was supported by the Kuratorium ZNS. We thank E. Thies, C. Scholz, and I. Meeßen for technical help, G. W. Eschweiler for help with the statistical analysis, and the Drs. R. Wingate, I. McKay, A. Goriely, and Y. von Boxberg for correcting the English and for critical discussions.

Correspondence should be addressed to Dr. Mathias Bähr, Neurologische Universitätsklinik, Hoppe-Seyler-Strasse 3, 72076 Tübingen, Germany.

Dr. Wizenmann's present address: Department of Anatomy, United Medical and Dental Schools, Guy's Hospital, London, UK.

Copyright (C) 1996 Society for Neuroscience $0270-6474 / 96 / 165106-11 \$ 05.00 / 0$
}

target regions, they showed a preference for the deafferented SC. On carpets consisting of laminin and membranes from normal SC (not deafferented) or nontarget regions (inferior colliculus), temporal and nasal axons grow either in a random fashion or show preferences for the laminin stripes.

Our modified version of the classic stripe assay shows specific growth preferences of embryonic retinal axons for membrane lanes from their appropriate embryonic or deafferented adult target regions. These findings suggest that the deafferentation of the SC in adult rats triggers the reexpression of specific guidance activities for retinal axons. Those "attractive" guidance cues appear to be differentially expressed in the developing and deafferented SC.

Key words: retina; retinotectal system; development; retinal ganglion cell; stripe assay; guiding cues
(Stahl et al., 1990; Drescher et al., 1995): one repulsive guidance molecule (Stahl et al., 1990) selectively affects the growth of temporal retinal axons, whereas a high dose expression of the other (Drescher et al., 1995) leads to the collapse of both temporal and nasal RGC growth cones.

A purified preparation of membranes revealed that nasal axons as well preferentially grow on membranes derived from posterior tectum, which is their natural target region (von Boxberg et al., 1993). It has been proposed that this could be because of a selective stabilization of nasal retinal axons by a trophic influence of posterior tectal membranes (von Boxberg et al., 1993).

Pioneer work suggests that guidance activities are only operating for a limited period of time during development and are downregulated after a specific projection has been formed (Walter et al., 1987a; Godement and Bonhoeffer, 1989). However, we have recently shown that putative guiding activities for regenerating retinal axons are reexpressed after deafferentation of the SC by optic nerve axotomy in adult rats (Wizenmann et al., 1993; Bähr and Bonhoeffer, 1994).

In the present study, we further describe the behavior of embryonic rat retinal axons on alternating stripes of laminin and membranes. Laminin was offered as an alternative growth substrate to membrane lanes prepared from embryonic, normal, or deafferented adult rat SC. This modification of the initial stripe assay was chosen to determine whether either adhesive/attractive or repulsive components dominate in brain regions of embryonic and adult rats and whether temporal and nasal retinal axons selectively grow on membranes from their specific target region.

\section{MATERIALS AND METHODS}

Microsurgical procedures. For optic nerve axotomy, adult female Sprague Dawley rats (200-300 gm) were deeply anesthetized with 7\% chloralhy- 
Table 1. Patterns of nasal axon growth on a substrate of membranes from different brain regions

A. Preferences of nasal axons on alternating stripes of SC (embryonic, deafferented, and adult) and laminin or inferior colliculus and laminin

\begin{tabular}{|c|c|c|c|c|c|}
\hline Brain region & & $\begin{array}{l}\text { No } \\
\text { preference }\end{array}$ & $\begin{array}{l}\text { Preference for } \\
\text { laminin }\end{array}$ & $\begin{array}{l}\text { Preference for } \\
\text { membranes }\end{array}$ & Significance \\
\hline \multirow[t]{2}{*}{ Embryonic SC } & $\mathrm{A}(n=12)$ & 4 & 5 & 3 & n.s. \\
\hline & $\mathrm{P}(n=12)$ & 1 & 3 & 8 & n.s. \\
\hline & $\mathrm{P}(n=20)$ & 4 & 0 & 16 & $p=0.01^{b}$ \\
\hline \multirow[t]{2}{*}{ Adult SC } & $\mathrm{A}(n=10)$ & 1 & 7 & 2 & n.s. \\
\hline & $\mathrm{P}(n=10)$ & - & 8 & 2 & n.s. \\
\hline
\end{tabular}

n.s., Not significant.

${ }^{a}$ Significant preference for laminin.

${ }^{b}$ Significant preference for membranes.

B. Nasal axons confronted with alternating stripes of deafferented and embryonic anterior or posterior SC membranes

\begin{tabular}{|c|c|c|c|c|}
\hline $\mathrm{SC}$ region & $\begin{array}{l}\text { No } \\
\text { preference }\end{array}$ & $\begin{array}{l}\text { Preference for } \\
\text { deafferented SC }\end{array}$ & $\begin{array}{l}\text { Preference for } \\
\text { embryonic SC }\end{array}$ & Significance \\
\hline Posterior $(n=30)$ & 5 & 19 & 6 & $p=0.01^{c}$ \\
\hline
\end{tabular}

n.s., Not significant.

${ }^{c}$ Note the significant preference for membranes from posterior deafferented SC.

drate $(420 \mathrm{mg} / \mathrm{kg}$ body weight, i.p.). The left optic nerve was unilaterally transected intraorbitally with iridectomy scissors immediately behind the eye as described previously (Bähr et al., 1988). Rats received a lethal dose of chloralhydrate at $14-60 \mathrm{~d}$ post-axotomy, and the right SC was removed. All surgical procedures were performed in agreement with state animal care laws and supervised by local authorities.

Membrane preparations. For membrane preparations (Walter et al., 1987a as modified by Wizenmann et al., 1993), embryonic (E16-E18), normal, deafferented adult rat superior, or adult rat inferior colliculi (14-60 d after contralateral optic nerve axotomy) was used. As shown previously, the guiding activities of deafferented colliculi are not signifi- cantly affected by time left for recovery. Thus, there was no difference in SCs 2 weeks or 2 months after optic nerve lesion (Wizenmann et al., 1993). Therefore, we pooled the colliculi of rats deafferented at different times before the membrane preparation. Carpets with alternating stripes of membranes and laminin on nuclepore filters were prepared as described recently (Wizenmann et al., 1993). Briefly, the filters were incubated for $2 \mathrm{hr}$ with laminin $[1 \mathrm{mg} / \mathrm{ml}$ in Hank's buffered salt solution (HBSS; BRL, Bethesda, MD)]. After preparation, dissociation, and centrifugation, the membrane fragments were resuspended in phosphate buffer, washed twice, and mixed with fluorescein isothiocyanate-labeled beads (Duke Scientific, Palo Alto, CA) to visualize their arrangement on

Table 2. Patterns of temporal axon growth on a substrate of membranes from different brain regions

A. Preferences of temporal axons on alternating stripes of SC (embryonic, deafferented, and adult) and laminin or inferior colliculus and laminin

\begin{tabular}{|c|c|c|c|c|c|}
\hline Brain region & & $\begin{array}{l}\text { No } \\
\text { preference }\end{array}$ & $\begin{array}{l}\text { Preference for } \\
\text { laminin }\end{array}$ & $\begin{array}{l}\text { Preference for } \\
\text { membranes }\end{array}$ & Significance \\
\hline \multirow[t]{2}{*}{ Embryonic SC } & $\mathrm{A}(n=12)$ & 1 & - & 11 & $p=0.001^{a}$ \\
\hline & $\mathrm{P}(n=12)$ & - & 12 & - & $p=0.009^{b}$ \\
\hline & $\mathrm{P}(n=20)$ & 14 & 1 & 5 & n.s. \\
\hline \multirow[t]{2}{*}{ Adult SC } & $\mathrm{A}(n=10)$ & 5 & 4 & 1 & n.s. \\
\hline & $\mathrm{P}(n=10)$ & - & 8 & 2 & n.s. \\
\hline
\end{tabular}

n.s., Not significant.

${ }^{a}$ Significant preference for membranes.

${ }^{b}$ Significant preference for laminin.

B. Temporal axons confronted with alternating stripes of deafferented and embryonic anterior or posterior SC membranes

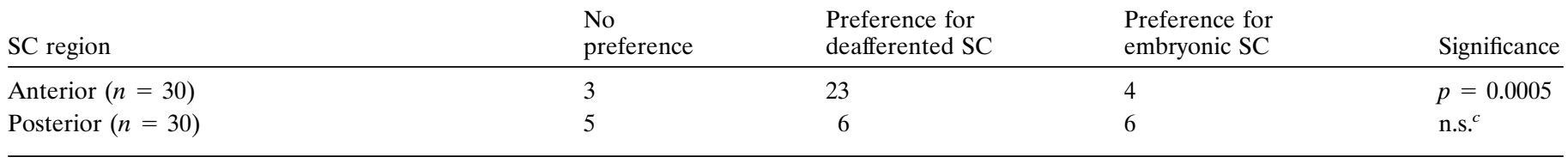

n.s., Not significant.

Note the significant preference for membranes from deafferented anterior SC.

${ }^{c}$ In that case, 13 of 30 explants did not show any outgrowth, suggesting an inhibitory effect for temporal axons by both deafferented and embryonic posterior membranes. 


\section{DEAFFERENTED}

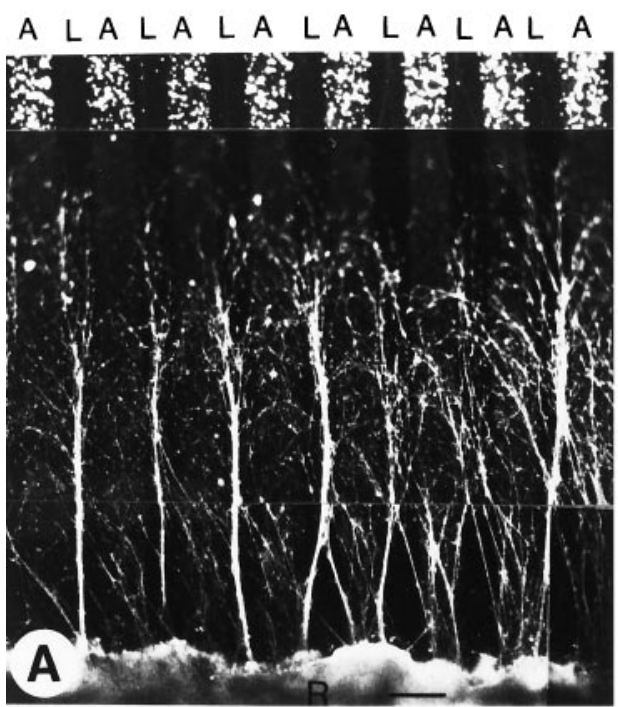

nasal
A L A L A L A L A L A L A L

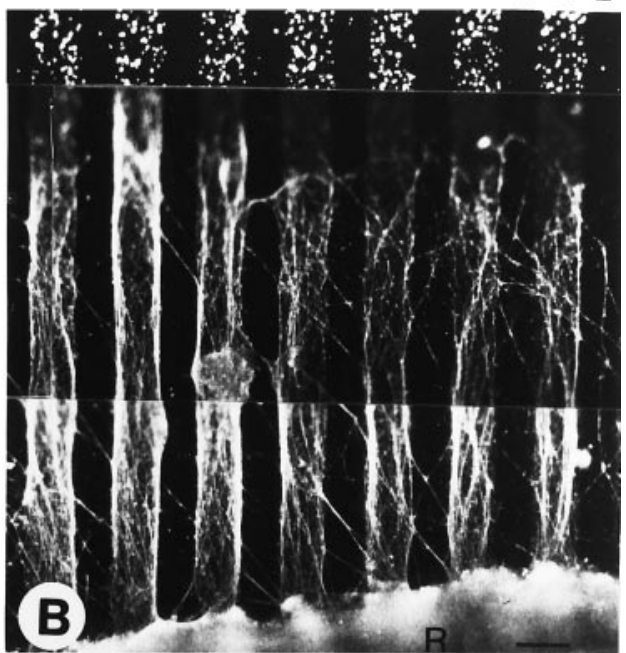

temporal

\section{NORMAL}

Figure 1. Axons of temporal rat retinae prefer membranes from deafferented anterior SC over laminin as substratum for growth. $A$, When striped carpets of laminin $(L)$ and membranes from deafferented anterior SC $(A$, indicated by a label with fluorescent beads on top of each panel) are prepared, most of the nasal retinal axons show clear preferences for the laminin lanes. Axons from the temporal retina on the same striped carpets preferentially grow on deafferented membranes from anterior SC $(B)$. On carpets with alternating stripes from normal adult anterior SC and laminin, temporal retinal axons show a random outgrowth $(C)$. When temporal retinal axons have the choice between membrane stripes from the inferior colliculus $(I)$ and laminin, however, they show a clear preference for the laminin lanes $(D)$. Retinal explants are indicated with $R$. Scale bar, $100 \mu \mathrm{m}$.

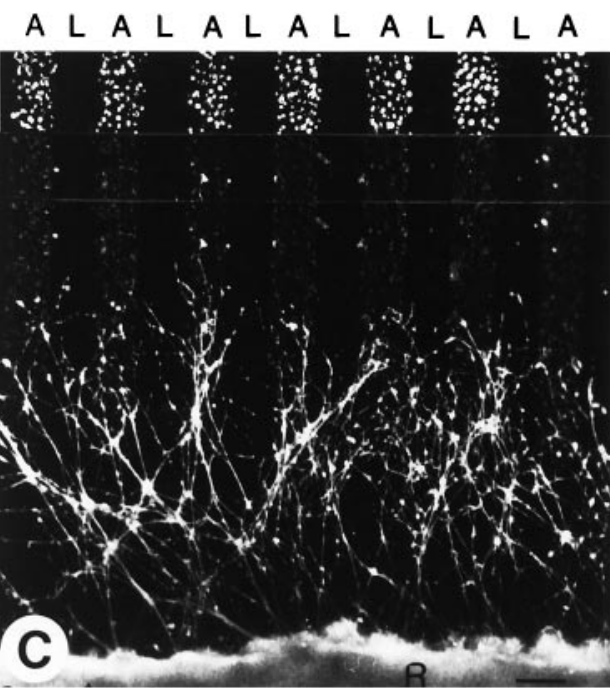

temporal

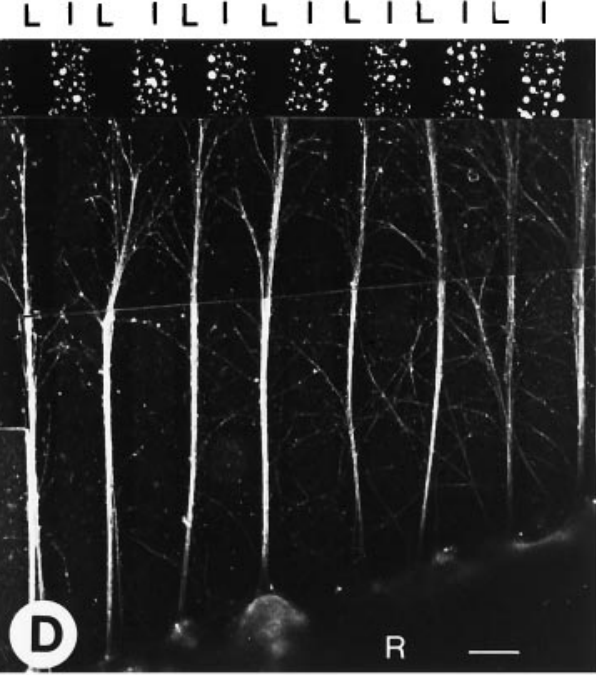

temporal the laminin-covered nuclepore filters. The membrane fraction was sucked on laminin-covered nuclepore filters in stripes. Thus, the carpet is composed of a repetition of a membrane lane underlain by laminin alternating with a pure laminin lane. For clarity, membrane lanes underlain by laminin will be referred to as membrane lanes. We believe that the way the membrane carpets are laid down makes the axons very unlikely to be influenced by the underlying laminin in their growth behavior on the membrane lanes. The conventional stripe assay with alternating membrane lanes was performed as described in Wizenmann et al. (1993).

Explantation and culturing of retina. Retinal explants were prepared as described previously (Wizenmann et al., 1993). Briefly, retinae were removed from rat embryos (E17-E19), freed from surrounding tissue, and whole-mounted on semipermeable nitrocellulose filters (Millipore, Bedford, MA). A suspension of the fluorescent dye Di-Asp (stock: 25 $\mathrm{mg} / 500 \mu \mathrm{l}$ of dimethylformamide; $15 \mu \mathrm{l}$ stock in $2 \mathrm{ml}$ of HBSS) was centrifuged onto the retinal explants to visualize RGC axons that had extended from the retinal explants. Stripes of retinae 250-300 $\mu \mathrm{m}$ wide were cut with a tissue chopper (Bachhofer, Reutinger, Germany) and explanted onto the membrane-laminin carpets. The embryonic rat retinae were grown in immunoglobulin-free, serum-containing medium (Life Technologies) for a reproducible detection of guidance activities in the SC membranes because, in an earlier study (Wizenmann et al., 1993), we found that the guidance activities were not detectable when media with a high immunoglobulin content were used. This might be attributable to the 
nasal

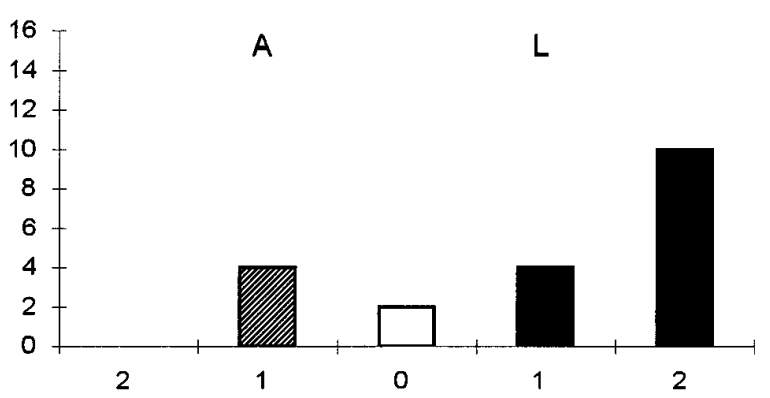

temporal

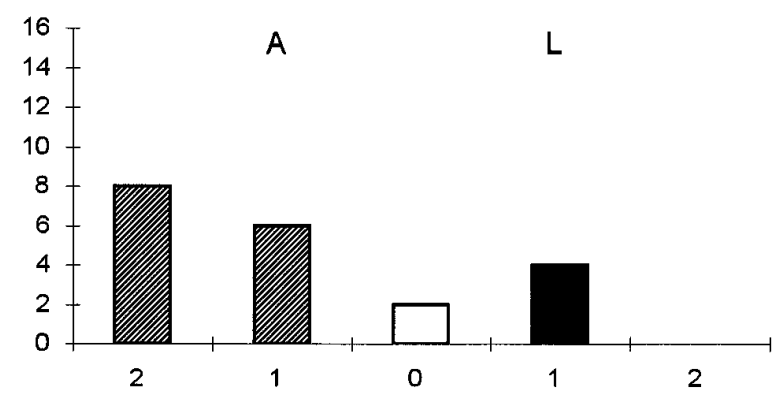

Figure 2. Growth preferences of retinal axons on alternating lanes with membranes from the deafferented anterior SC and laminin. The figure shows the growth behavior of axons from nasal and temporal retinal explants on striped carpets with membranes of the deafferented anterior SC and laminin. Axons from nasal retinal explants prefer to grow on laminin lanes. Axons from temporal retinal explants show a significant preference for membranes from the deafferented anterior SC over laminin. The ordinate shows the numbers of retinal explants, the abscissa the growth preferences for each substrate. $A$, Anterior SC membranes; $L$, laminin.

interference of certain types of immunoglobulins with the respective guidance molecules and might especially apply to attractive guidance cues (Bähr et al., unpublished observations).

Analysis of axon growth on membrane carpets. The analysis of axonal growth was performed as a double-blind experiment by two independent observers. Each observer recorded the axon density on anterior and/or posterior membrane stripes. As discussed previously (Vielmetter and Stuermer, 1989; Vielmetter et al., 1991; Wizenmann et al., 1993), it was impossible to count individual retinal axons because of axon fasciculation and the massive outgrowth. Therefore, we judged the growth preferences of the neurites using a three-class system: no preference or random growth (0), slight and moderate (1), and clear-cut preference (2). Walter et al. (1987a) defined a four-class system to judge growth preferences of axons. They distinguished between slight and moderate preference. The inter-rater reliability in our experiments was not high enough to allow us to make a reproducible distinction between slight and moderate preferences. The data from individual experiments were pooled, and chi-square tests were performed. The inter-rater reliability was $>90 \%$ and did not vary significantly in the different experimental settings.

To determine the overall extent of elongation of retinal axons on membranes from different regions (anterior and posterior SC, IC, or laminin), the distances between the front of the longest neurites and the retinal explant were measured in three experiments after $2 \mathrm{~d}$ of growth in vitro. The mean rate of axon growth was determined by dividing the longest extension of the neurite front by the time spent in vitro $(48 \mathrm{hr})$ and expressed in micrometers per hour. For statistical analysis, $t$ tests were performed.

\section{RESULTS}

\section{A modified stripe assay allows one to distinguish attractive versus repulsive guidance activities in vitro}

Our goal was to examine whether attractive and/or repulsive guidance activities within membrane preparations from embryonic and adult (normal and deafferented) SC cells play a role in guiding retinal axons in vitro. To test this hypothesis, we modified the stripe assay system developed by Walter et al.(1987a) and used alternating lanes of laminin and membranes, instead of alternating lanes of different membrane fractions. A similar modification of the stripe assay has been successfully used by Bastmeyer and Stürmer (1993) for a time-lapse study in the fish retinotectal system.

Strictly speaking, the membrane lanes are underlain by laminin. However, for the sake of simplicity and because it is very unlikely that they could influence the behavior of axons growing on the membranes, we will refer to them as membrane lanes only. As laminin supports extensive growth of retinal axons, the presence of repulsive components within membrane lanes is assessed by a preference of the retinal axons for the laminin lanes. In contrast, attractive or adhesive cues within membranes result in either a random outgrowth, which suggests that there is no preference for one of the lanes, or in axons growing preferentially on the membrane lane, in which case the attractive cues are stronger than the effect of laminin.

Explants from embryonic rat retinae were used to determine the growth preferences. As shown previously, embryonic rat and chick RGC axons are able to detect positional cues present in the embryonic and deafferented adult rat SC (Wizenmann et al., 1993). Adult retinae were not used in the present experiments, because it has been shown that their RGC axons do not grow out consistently in the stripe assay, possibly attributable to the presence of myelin-associated inhibitors in membrane fractions $(\mathrm{Ca}-$ roni and Schwab, 1988; Bähr and Schwab, 1996). The focus of the present study is the examination of guidance activities present or reexpressed in the adult SC after deafferentation.

To study the positional cues involved in axon guidance, embryonic rat axons from nasal and temporal retina were grown on alternating stripes of laminin and membranes derived from anterior or posterior deafferented SC (section 1), embryonic SC (section 2), normal SC (not deafferented), or inferior colliculus (section 4). In addition, temporal and nasal retinal axons were grown on embryonic anterior and deafferented anterior or embryonic posterior and deafferented posterior SC membranes (section 3). We scored the growth behavior of retinal axons using a three-class system (see Materials and Methods; Wizenmann et al., 1993). Finally, to determine the rate of axon growth, the average length of the retinal axons on membranes or on laminin alone was determined (section 5).

\section{Section 1. Axonal growth behavior on alternating stripes of laminin and membranes from deafferented target regions}

When given the choice between membranes from anterior SC and laminin, the majority of nasal axons $(n=20$; Table 1A) showed a moderate or clear-cut preference for laminin lanes (14 of 20; Figs. $1 A, 2$, Table 1). In two explants, no growth preference was noted, and in four explants the axons grew preferentially on the membrane lanes. Axons from temporal retina $(n=20$; Table 2$)$ exhibited a strong preference for membrane lanes from deafferented anterior SC, their target region (14 of 20; Figs. $1 B, 2$ ). Both of these preferences are statistically significant (chi-square test; Tables 1, 2).

When nasal retinal explants $(n=20$; Table $1 \mathrm{~B})$ were placed on 


\section{DEAFFERENTED}

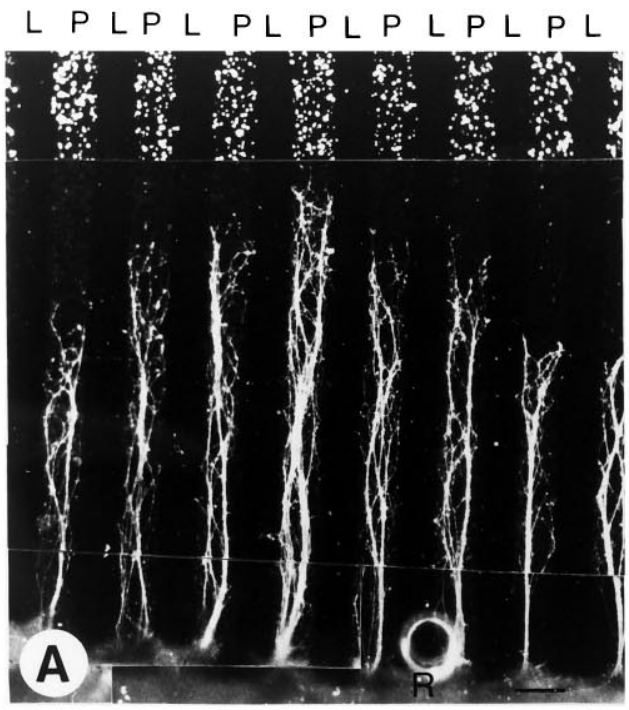

nasal

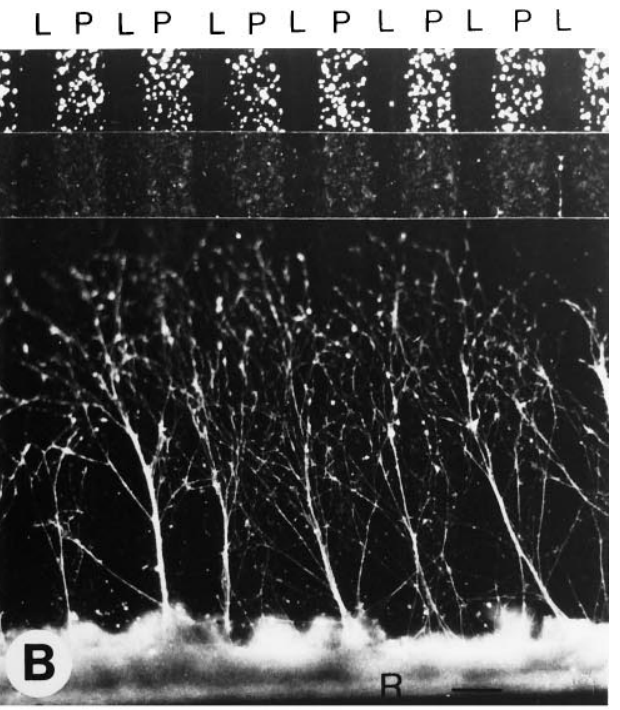

temporal

\section{NORMAL}

Figure 3. Axons of nasal rat retinae prefer membranes from deafferented posterior SC over laminin as substratum for growth. On striped carpets consisting of laminin $(L)$ and membranes from deafferented posterior SC (indicated by $P)$, nasal retinal axons show a strong preference for the membranes from deafferented posterior SC $(A)$, but temporal retinal axons $(B)$ on the same carpets show a random growth pattern. Axons from nasal retinal explants cultured on alternating stripes from normal adult posterior SC [not deafferented, deafferented posterior $(P)$, and laminin $(L)]$ grow preferentially on the laminin lanes (C). In stripe assays with membranes from the inferior colliculus $(I)$ and lami$\operatorname{nin}(L)$, nasal retinal axons also grow preferentially on the laminin lanes $(D)$. Retinal explants are indicated with $R$. Scale bar, $100 \mu \mathrm{m}$.
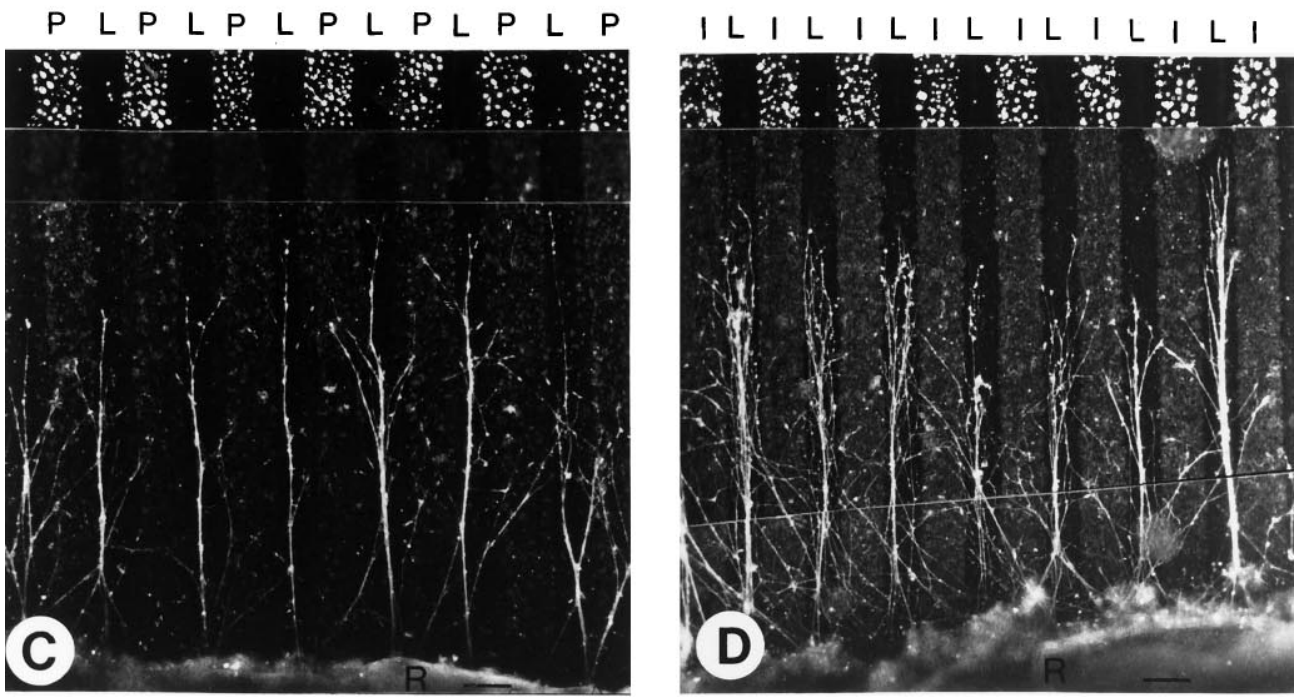

nasal nasal alternating stripes with membranes from deafferented posterior $\mathrm{SC}$ and laminin, their axons clearly preferred the membrane lanes derived from their appropriate target region, the posterior SC (16 of 20 clear-cut decision; Figs. $3 A, 4)$. The preference of axons from nasal retinal explants for stripes from the deafferented posterior SC is statistically highly significant. Axons from the temporal retinal explants $(n=20)$ grown on the same type of carpets showed a random outgrowth in 14 of 20 explants (Figs. $3 B$, 4) and a tendency to grow on membranes rather than on laminin in only five cultures (Table 2A).
These results clearly indicate that temporal and nasal retinal axons prefer growing on membranes derived from their specific target regions than on laminin.

Section 2. Outgrowth of axons from temporal and nasal retina on alternating stripes of laminin and membranes from embryonic SC

To assess the preferences of temporal and nasal RGC axons for their target, they were offered alternating lanes of embryonic rat $\mathrm{SC}$ and laminin ( $n=12$ each). 
nasal

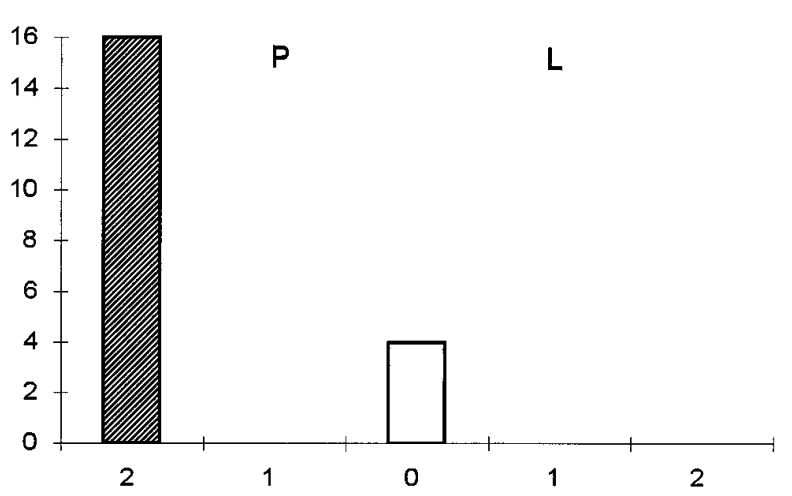

temporal

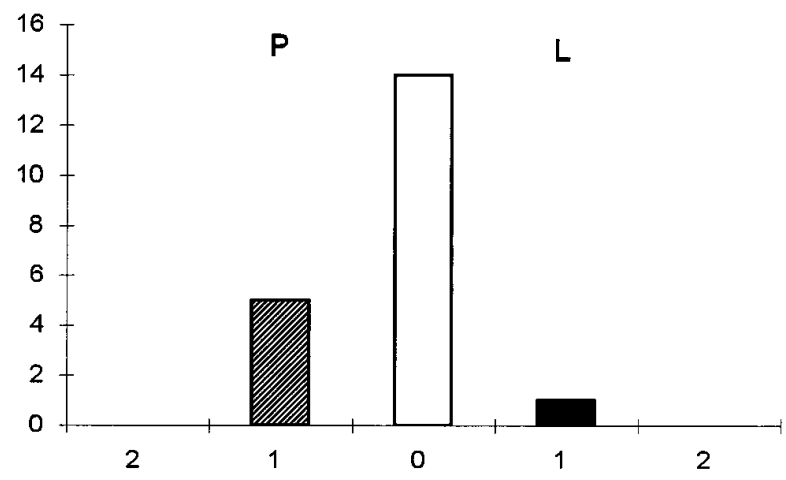

Figure 4. Growth preferences of retinal axons on alternating lanes with membranes from the deafferented posterior SC and laminin. The figure shows the preferences of axons from temporal and nasal retinal explants on striped carpets consisting of laminin and membranes from deafferented posterior SC. Axons from nasal retinal explants show a significant preference for membrane lanes, whereas temporal retinal axons show no significant growth preference. The ordinate shows the numbers of retinal explants, the abscissa the growth preferences for each substrate. $P$, Posterior SC membranes; $A$, anterior SC membranes; $L$, laminin.

When stripes of posterior SC membranes are juxtaposed to laminin stripes, temporal axons always preferred to grow on laminin (Table 2A). In contrast, nasal axons show a clear-cut or moderate preference ( 8 of 12 cases) for posterior SC membranes (Fig. 5; Table 1A). Although this result suggests a preference for the membranes from posterior embryonic SC, it is not statistically significant, because in three explants a preference for laminin was observed.

When grown on alternating lanes of membranes from embryonic anterior SC and laminin, nasal axons showed no preference for either substrate (Fig. 5, Table 1A). In contrast, axons from temporal retinal explants showed a clear-cut preference for membranes from anterior SC (11 of 12), a highly statistically significant preference of temporal axons for anterior membranes. (Fig. 5, Table 2A).

Thus, temporal and nasal retinal axons seem to prefer to grow on membranes from their specific embryonic target region over laminin. However, statistical analysis reveals that only the choice of temporal, but not of nasal, retinal axons for membranes from its specific target region is significant.

\section{Section 3. Axonal growth behavior on alternating membrane} stripes from embryonic and deafferented adult rat $S C$

To evaluate which targets (adult or embryonic) exert a stronger effect on retinal axons, alternating stripes from the same region of the embryonic and adult rat $\mathrm{SC}$ were used as substrate for retinal explants. RGC axons from nasal retina $(n=30)$ preferred growing on membranes from deafferented posterior SC over that of embryonic posterior SC: 19 of 30 nasal explants exhibited a moderate (6) or a clear-cut (13) preference for the adult deafferented membranes, a result statistically significant. In only 6 of 30 explants did nasal axons grow preferentially on stripes derived from posterior embryonic SC (Fig. 6, Table 1B).

On the same type of membrane carpets, temporal axons showed no growth preference (5 of 30 explants). In six cases, the embryonic membrane lanes were preferred, and in another six cases the adult membrane lanes were preferred. However, 13 explants showed no outgrowth at all (Fig. 6, Table 2B). This was never observed in any other experimental condition. When nasal axons were confronted with alternating stripes from embryonic and adult deafferented anterior SC, no growth preference was ob- served (15 of 30 explants). A moderate preference for deafferented anterior membranes was noted in eight cases, and seven explants moderately preferred to grow on embryonic membranes (Fig. 6, Table 1). Axons from temporal retinal explants showed a statistically significant preference for membranes from deafferented anterior SC (23 of 30; Fig. 6, Table 2).

These results show that retinal axons prefer membranes derived from deafferented versus embryonic SC only if the membranes originate from their appropriate target region (topographic match).

\section{Section 4. Axonal growth behavior on alternating stripes of laminin and membranes from normal SC and nontarget regions}

In control experiments, retinal explants were placed on alternating stripes consisting of membranes prepared from normal adult $\mathrm{SC}$ and laminin. Nasal retinal axons showed a slight preference for membranes from normal posterior SC in only two cases $(n=10$; Figs. $3 C, 7)$. The rest of the nasal explants grew on laminin (8 of 10). Similarly, temporal axons preferred the laminin lanes in 8 of 10 explants. However, two explants grew preferentially on the membrane lanes (Fig. 7). Statistically, however, the preferences of the nasal and temporal axons for the laminin lanes are not significant.

When offered alternating lanes of laminin and membranes from normal anterior SC, temporal retinal explants did not show any growth preference ( $n=10$; Figs. $1 C, 7$, Table 1$)$. By contrast, the same experimental condition leads to a clear preference of nasal fibers for laminin lanes ( 7 of 10; Fig. 7, Table 1). However, a clear and statistically significant preference of both temporal $(n=10$; Figs. $1 D, 8)$ and nasal $(n=10$; Figs. $3 D, 8)$ retinal axons for laminin lanes was observed when membranes derived from the inferior colliculus were offered as a choice.

Taken together, the control experiments show that temporal and nasal retinal axons clearly prefer growing on laminin than on membrane lanes from normal adult SC of inferior colliculus.

\section{Section 5. Extent of axonal elongation on membrane} preparations from the deafferented adult rat $S C$ and laminin

To further evaluate the substrate properties, the maximal extent of elongation of the axons from nasal and temporal retinal ex- 


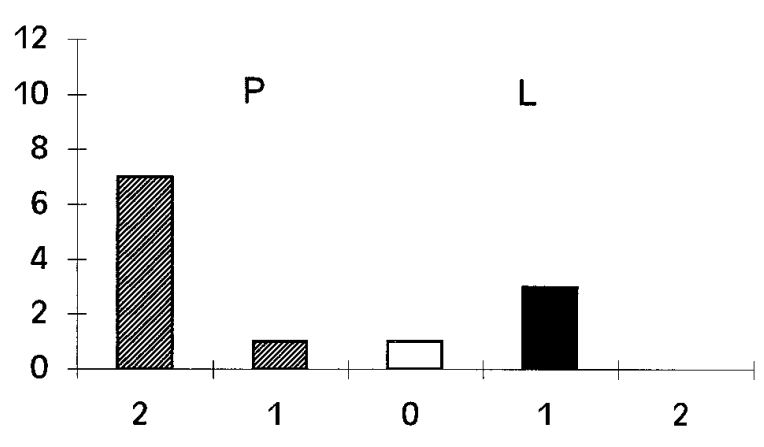

nasal

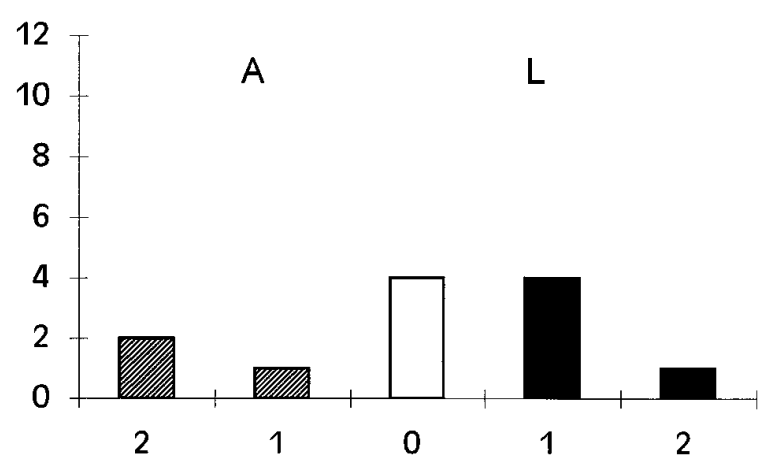

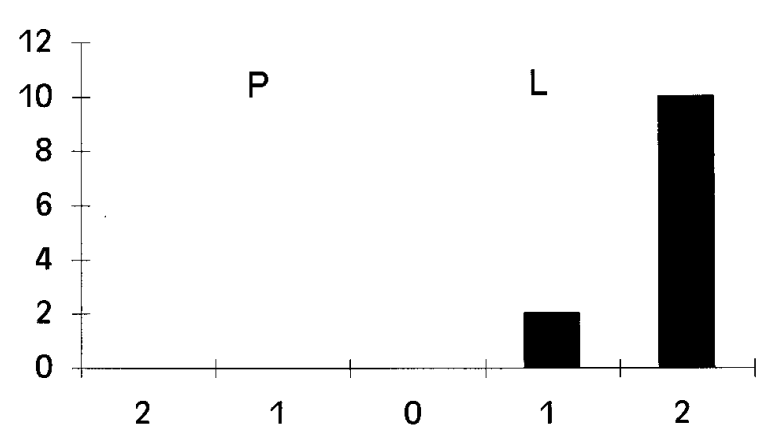

temporal

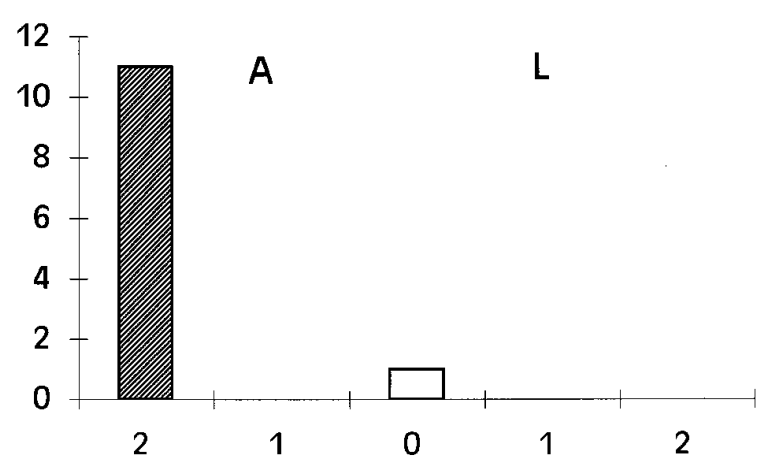

Figure 5. Growth preferences of retinal axons on alternating lanes with membranes from the embryonic SC and laminin. The figure shows the growth preferences of retinal axons on striped carpets with membranes from the embryonic SC and laminin. On striped carpets with membranes from embryonic posterior SC and laminin, axons from nasal retinal explants showed preferences for the membrane lanes, although this was not a statistically significant preference for membrane lanes from the posterior part of the embryonic SC. On alternating stripes of embryonic posterior SC membranes and laminin, axons from temporal retinal explants always preferred to grow on the laminin lanes. On carpets with membranes from embryonic anterior SC and laminin, axons from nasal retinal explants showed no significant preference for either membrane or laminin lanes. In contrast, axons from temporal retinal explants showed a significant preference for membrane lanes from anterior SC over laminin. The ordinate shows the numbers of retinal explants, the abscissa the growth preferences for each substrate. $P$, Posterior SC membranes; $A$, anterior SC membranes; $L$, laminin.

plants was determined for different conditions of the stripe assay $(n=59$; Fig. 9). Axons from temporal retinal explants grew at a mean rate of $12.2 \pm 2.5 \mu \mathrm{m} / \mathrm{hr}$ on deafferented anterior membranes. On laminin, RGC axons from temporal retinal explants grew at a rate of $21.0 \pm 4.6 \mu \mathrm{m} / \mathrm{hr}$. Nasal axons exhibited a growth rate of $11.3 \pm 5.4 \mu \mathrm{m} / \mathrm{hr}$ on deafferented posterior membranes and $14.8 \pm 1.5 \mu \mathrm{m} / \mathrm{hr}$ on laminin lanes. These growth rates of axons from nasal and temporal retinal explants on membranes of their appropriate target region are not significantly different from one another. When retinal axons grew preferentially on laminin lanes, however, they do so faster than on the membrane substrate $(t$ test, $p<0.05)$.

\section{DISCUSSION}

In principle, axon guidance toward or within target regions can be accomplished both by repulsive mechanisms that prevent ingrowth into nontarget regions or by attractive cues presented by the target region. In the developing retinotectal system, two repulsive guidance molecules, RAGS (Drescher et al., 1995) and RGM (Stahl et al., 1990), have been described. In contrast, the existence of attractive guidance cues in this system is less well established.
For birds and mammals, the formation of nerve connections in the CNS takes place within a certain time window. Adult CNS tissue does not support axon growth, rendering regeneration of lesioned connections impossible. Furthermore, the guidance activities for growing axons, present during development, are thought to be absent in adult brain (Walter et al., 1987a). However, it has recently been shown that under certain circumstances axons from adult brain can be induced to regenerate and regrow toward their natural target (Bähr and Eschweiler, 1991, 1993; Hankin and Lund, 1991).

We are interested in understanding whether regenerating axons are capable of establishing ordered functional connections within their normal target areas. This would imply the reexpression of guidance molecules, which are normally active only during development. Our goal was to confirm and extend previous results (Wizenmann et al., 1993) demonstrating that guidance mechanisms can indeed be reactivated in adult brain. We show, using an in vitro assay, that retinal axons appear to recognize specifically cell membranes derived from their respective target area in both embryonic and adult SC. A prerequisite for recognition of the adult target region, however, was its deafferentation at least 2 

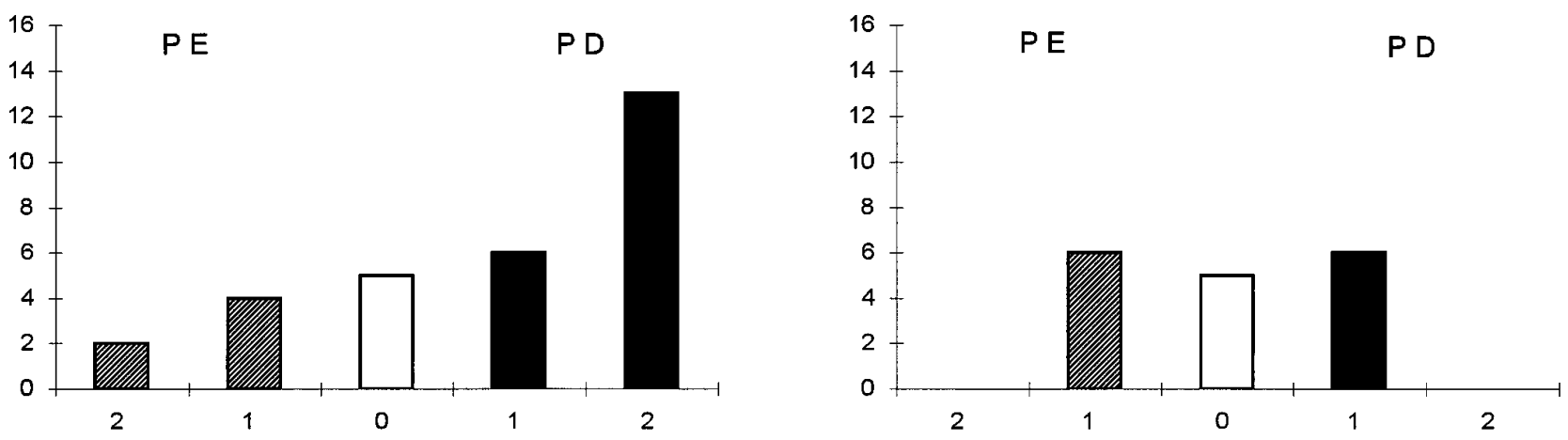

nasal

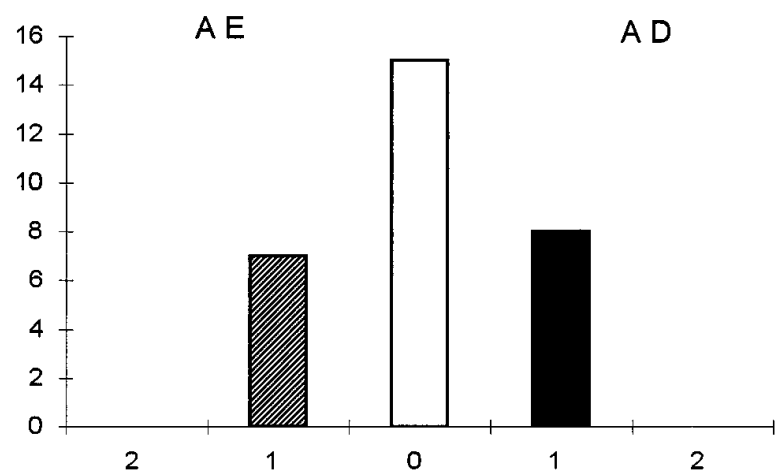

temporal

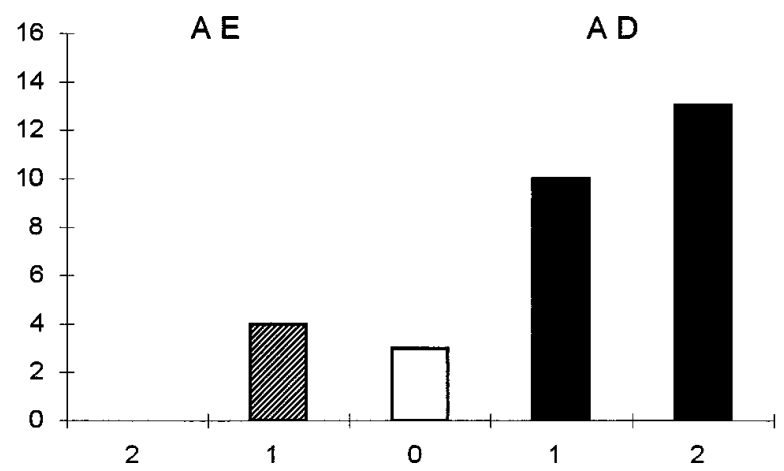

Figure 6. Growth preferences of retinal axons on alternating lanes with membranes from the embryonic and adult deafferented SC. When grown on alternating lanes with membranes from embryonic and adult deafferented posterior SC, axons from nasal retinal explants showed significant growth preferences for the membranes from deafferented adult posterior SC. Axons from temporal retinal explants in these cultures showed no statistically significant preference for either lanes with membranes from embryonic or lanes with membranes from deafferented adult posterior SC. Outgrowth was usually bad in these cultures. On alternating stripes from embryonic and deafferented adult anterior SC, axons from nasal retinal explants show no statistically significant preference for either stripes from embryonic or deafferented anterior SC. Axons from temporal retinal explants show clear growth preferences for lanes from deafferented adult anterior SC. The ordinate shows the numbers of retinal explants, the abscissa the growth preferences for each substrate. $P$, Posterior SC membranes; $A$, anterior SC membranes; $E$, embryonic; $D$, deafferented.

weeks before performing the test. Our results suggest that attractive and/or adhesive activities are upregulated in deafferented SC and that retinal axons can recognize these cues.

The assay system we applied to detect guidance cues in the deafferented SC, is the stripe assay (Walter et al., 1987a), which had been developed originally to analyze guidance activities in the developing retinotectal projection (Walter et al., 1987b). We used embryonic retinal explants, both for technical reasons and because we wanted to focus on guidance molecules in the SC. In our previous study, we had already noticed a slight difference in the outcome of the stripe assay when using either embryonic or adult deafferented SC membranes: in the stripe assay performed with embryonic SC membranes, temporal axons show a clear-cut preference for anterior SC, very likely attributable to the expression of repulsive guiding molecules in the posterior SC (Stahl et al., 1990; Drescher et al., 1995). Nasal axons, however, exhibit a similar preference for their respective target, the posterior SC, only after special treatment of the membranes (von Boxberg et al., 1993). In the case of the deafferented adult SC membranes, the order of preference seemed reversed. It was the nasal fibers that showed a more clear-cut preference for posterior SC than the temporal fibers for anterior SC (Wizenmann et al., 1993).
Here, we modified the original stripe assay for two reasons. First, to assess the above-mentioned effect further and to quantify it using statistical analysis. Second, to be able to better discriminate between the different adhesive, attractive, and repulsive mechanisms potentially active in retinal axon pathfinding. In this modified stripe assay, retinal axons have the choice to grow either on lanes of laminin alone, or laminin plus one of the following seven membrane types: adult normal anterior and posterior, adult deafferented anterior and posterior, embryonic anterior and posterior SC, and normal IC. Laminin appears to be a good supporting substrate for outgrowth of embryonic rat and chick retinal axons (Cohen et al., 1987). Consistent with this, retinal axons grow faster on pure laminin than on laminin plus one of the SC membrane types (see section 5 in Results).

The results we obtained with this modified stripe assay argue for attractive or adhesive cues being reexpressed in deafferented $\mathrm{SC}$ for the following reasons:

1. Nasal and temporal fibers clearly preferred the deafferented $\mathrm{SC}$ membranes of their natural target region when they were given the choice between deafferented SC membranes and pure laminin.

2. This preference was not observed for membranes from non- 
nasal

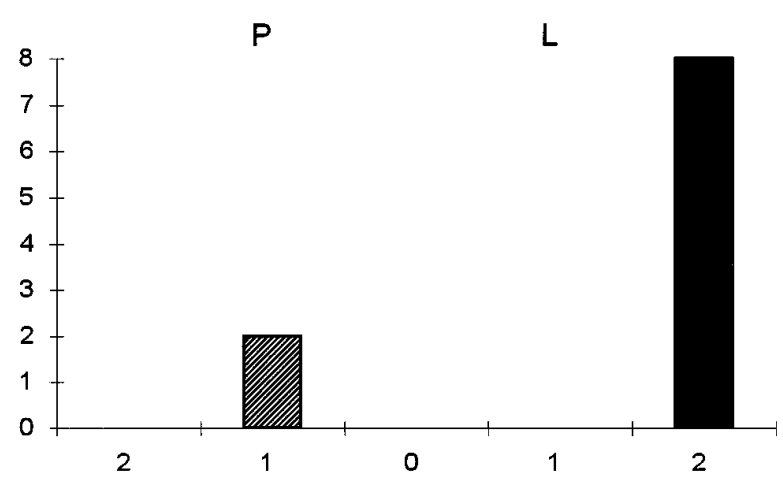

nasal

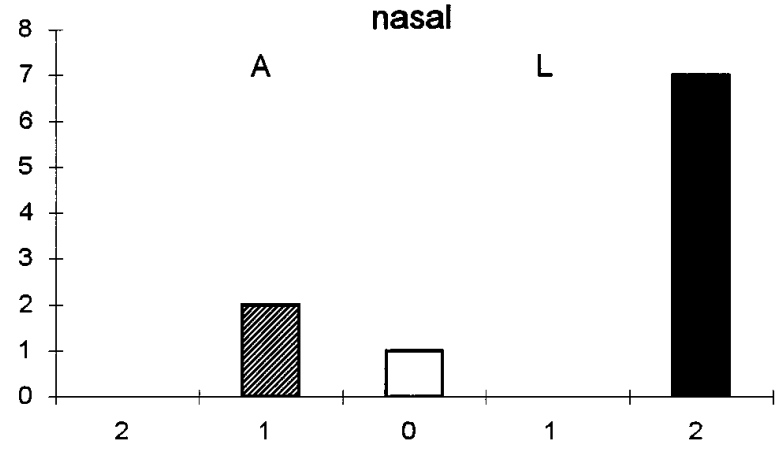

temporal

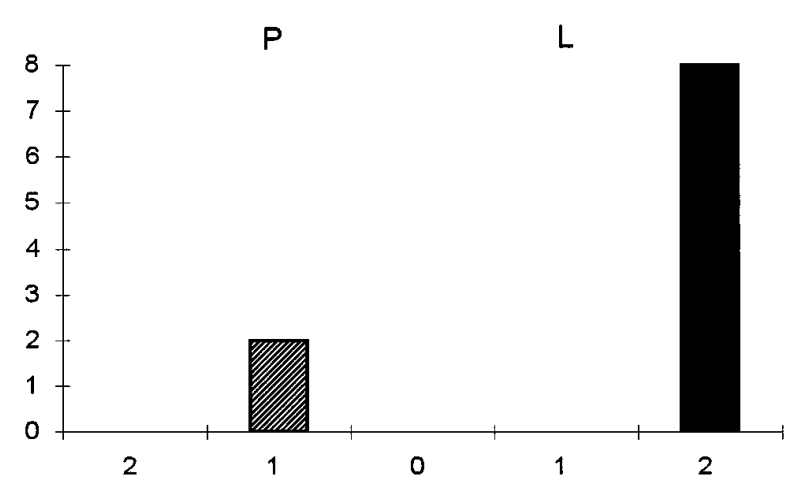

temporal

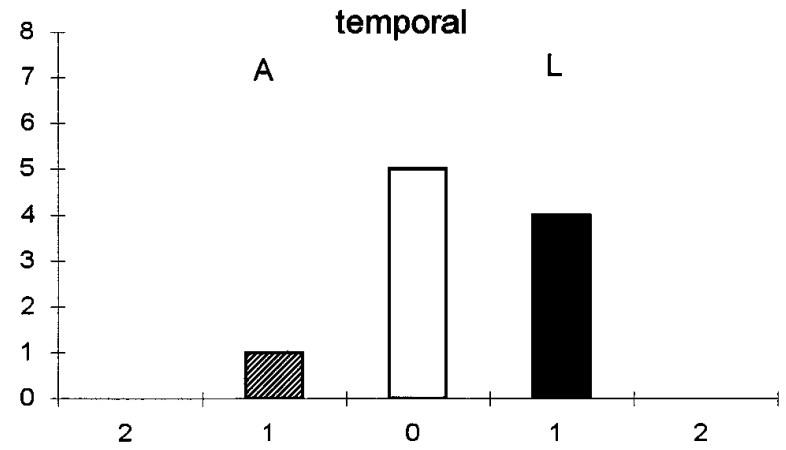

Figure 7. Growth preferences of retinal axons on alternating lanes with membranes from the normal adult rat SC and laminin. On carpets with alternating stripes from normal adult posterior SC and laminin, nasal and temporal retinal axons prefer to grow on laminin lanes. On carpets with membranes from normal adult anterior SC and laminin, axons from temporal retinal explants show a random growth pattern, whereas axons from nasal retinal explants show a preference for laminin stripes. The ordinate shows the numbers of retinal explants, the abscissa the growth preferences for each substrate. $P$, Posterior SC membranes; $A$, anterior SC membranes; $L$, laminin.

target regions: retinal axons grew preferentially on the laminin lanes when the juxtaposed membranes were derived from posterior nondeafferented SC or IC. A random outgrowth of retinal fibers was observed when membranes from the anterior nondeafferented SC were alternating with laminin lanes.

3. The preference of retinal axons to grow on membranes from deafferented SC, despite their higher speed of growth on laminin alone, strongly suggests the presence of specific tropic (attractive) cues. Interestingly, in vivo, axons slow down in their growth speed immediately before reaching their final target (Jhaveri, 1991). Furthermore, this growth preference for adult deafferented SC membranes should be specific because it is not seen with membranes derived from other brain regions, and because with certain combinations, fibers also prefer pure laminin over laminin plus deafferented SC membranes.

Moreover, our results suggest that especially attractive cues are present in the adult deafferented SC, and are even more prominent than in the developing SC. Both temporal and nasal retinal axons prefer to grow on membranes derived from their specific target region of the deafferented SC, whereas only temporal retinal explants showed a statistically significant preference for their embryonic target region, the anterior SC.

In contrast to the expression of attractive cues for nasal fibers, expression of repulsive guidance molecules for temporal axons in the posterior SC of adult rats after optic nerve lesion seems to be less potent than during development. This is based on the observation that temporal axons did not always avoid membranes from posterior deafferented SC (Wizenmann et al., 1993) and that a clear repulsion should have been seen in the modified stripe assay, where temporal axons have the choice to grow on laminin instead of posterior membranes (see section 1 in Results). The fact that outgrowth of temporal retinal axons from retinal explants was completely inhibited in 13 of 20 explants, where posterior membranes from embryonic and adult deafferented SC were presented as alternating lanes, suggests that a considerable amount of inhibitory activity remains present or is reexpressed in the posterior membranes after deafferentation (see section 4 in Results).

There are several different explanations for the preference of nasal axons being stronger for adult deafferented then for embryonic posterior SC. First, it is possible that during embryogenesis, posterior SC cells are induced to express certain guidance cues only after ingrowth of nasal fibers, which they will nevertheless be able to memorize after deafferentation. Second, specific trophic factors will be reexpressed after deafferentation, which during embryogenesis of the system come into play rather late (Hankin and Lund, 1991). Then, the growth substrate molecules presented by adult deafferented SC membranes may well be different from those expressed in developing SC, which could in turn affect the reaction of retinal axons to certain guidance cues. Finally, guidance cues active during embryogenesis and in the regeneration 
nasal

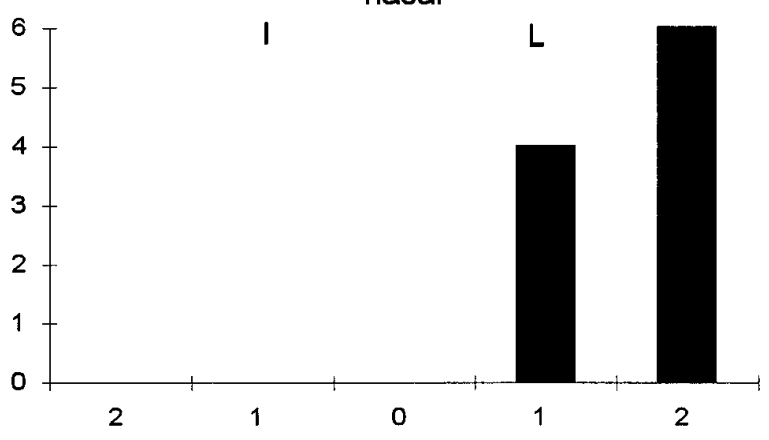

temporal

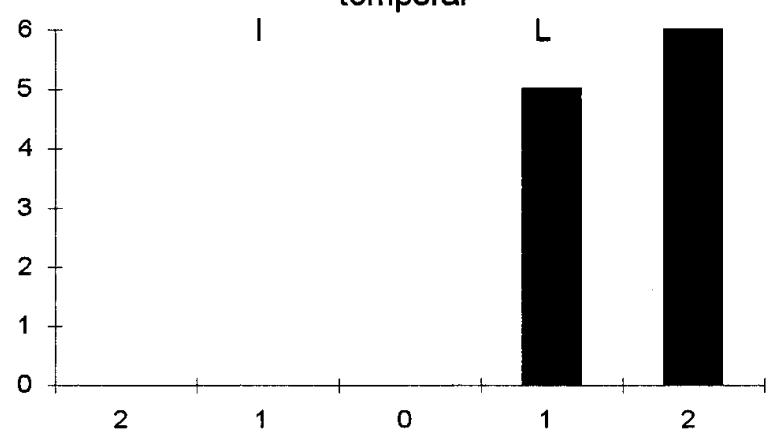

Figure 8. Growth preferences of retinal axons on alternating lanes with membranes from the inferior colliculus and laminin. When axons from temporal retinal explants are given the choice between membrane stripes from the inferior colliculus and laminin, they show a clear preference for the laminin lanes. Axons from nasal retinal explants also show a significant preference for the laminin lanes. The ordinate shows the numbers of retinal explants, the abscissa the growth preferences for each substrate. I, Inferior colliculus; $L$, laminin.

process need not be the same. In previous experiments (Wizenmann et al., 1993), the removal of putative repulsive factors by phosphatidylinositol phospholipase C (PI-PLC) treatment from membranes derived from deafferented SC did not lead to a random outgrowth of temporal fibers as had been shown for embryonic chick tectal membranes (Walter et al., 1987b), where this treatment removed "repulsive guidance molecules," which are GPI-anchored (Stahl et al., 1990; Drescher et al., 1995). PI-PLC treatment of membranes from anterior or posterior deafferented SC obviously removed not only putative repulsive factors but also growth-supporting components. Thus, temporal and nasal axons preferentially grew on the untreated membrane lanes, even of a type they would normally avoid (temporal axons on posterior membranes). When both membrane fractions (anterior and posterior) from adult deafferented SC were incubated with the enzyme, neither temporal nor nasal retinal axons showed any growth preference (Wizenmann et al., 1993).

The present results indicate that the reexpression of guidance factors after deafferentation is more complex than a simple recapitulation of their normal developmental. At this stage, we are not

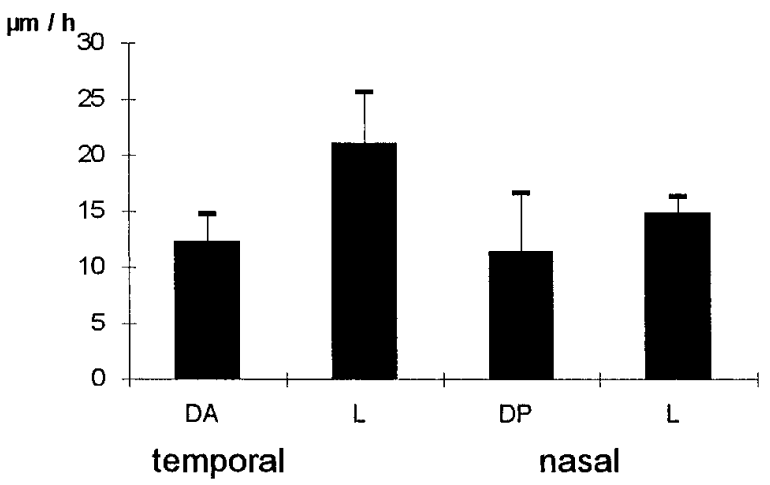

Figure 9. Extent of axonal elongation on membrane preparations from the deafferented adult rat SC and laminin. The figure shows the overall rates of elongation of nasal and temporal retinal axons on membranes and laminin. Axons from temporal retinal explants grew at a mean rate of $12.2 \pm 2.5 \mu \mathrm{m} / \mathrm{hr}$ on deafferented anterior membranes $(D A)$. On laminin $(L)$, axons from temporal retinal explants grew at a rate of $21.0 \pm 4.6$ $\mu \mathrm{m} / \mathrm{hr}$. RGC axons from nasal retinal explants grew at a rate of $11.3 \pm 5.4$ $\mu \mathrm{m} / \mathrm{hr}$ on deafferented posterior membranes $(D P)$ and at a rate of $14.8 \pm$ $1.5 \mu \mathrm{m} / \mathrm{hr}$ on laminin lanes. The growth rates of nasal and temporal axons on the membranes of their appropriate target regions are not significantly different. able to assess the nature of this difference, i.e., whether it is qualitative or merely quantitative. Such an understanding will require identification of the molecular nature of the specific guidance molecules in the retinocollicular system in the rat. So far, our results clearly show that in addition to the repulsive activities that have been identified, retinal axons can indeed be guided by attractive cues.

The existence of attractive cues has been substantiated by experimental evidence in several tissue culture systems involving peripheral nervous system and CNS tissue (for review, see Kennedy and Tessier-Lavigne, 1994). These factors may, in addition or alternatively, lead to a selective stabilization of axon terminals in vivo (Simon and O'Leary, 1992a,b; Roskies and O'Leary, 1994; Simon et al., 1994). The observations made with the modified version of the stripe assay presented here or with the "crossed version" of the stripe assay used by O'Leary and coworkers (Roskies and O'Leary, 1994) both suggest that the combination of repulsive and adhesive or attractive factors work together in establishing a topographic order in the retinotectal system.

In summary, the results of the present study indicate that both inhibitory and attractive cues are likely to guide retinal axons in vitro. In deafferented adult CNS, target structures, especially trophic/attractive components, could account for the plastic changes that occur after lesions. Together with the recent findings showing that adult CNS neurons can survive axotomy and regenerate axons that are able to establish functional contacts with specific target cells in vitro (Bähr and Eschweiler, 1991, 1993) and in vivo (Hankin and Lund, 1991), the results presented here suggest that complicated topographically ordered projections such as the retinotectal system may be restored after lesions. Thus, the mammalian CNS seems to be able to reactivate programs necessary for specific axon guidance and targeting in a manner similar to the situation described in fish and amphibians. Thus, there is hope that basic functions of the adult mammalian CNS may be regained if inhibitory aspects of the adult CNS environment are either neutralized or replaced by growth-permissive pathways.

\section{REFERENCES}

Bähr M, Bonhoeffer F (1994) Perspectives on axonal regeneration in the mammalian CNS. Trends Neurosci 17:473-479.

Bähr M, Eschweiler GW (1991) Regenerating adult rat retinal axons reconnect with target neurons in vitro. NeuroReport 2:581-584.

Bähr M, Eschweiler GW (1993) Formation of functional synapses by regenerating adult rat retinal ganglion cell axons in midbrain target regions in vitro. J Neurobiol 24:456-473. 
Bähr M, Schwab M (1996) Antibody that neutralizes myelin-associated inhibitors of axon growth does not interfere with recognition of targetspecific guidance information by rat retinal axons. J Neurobiol, in press.

Bähr M, Vanselow J, Thanos S (1988) In vitro regeneration of adult rat ganglion cell axons from retinal explants. Exp Brain Res 73:393-401.

Bastmeyer M, Stuermer CAO (1993) Behaviour of fish retinal growth cones encountering chick caudal tectal membranes: a time-lapse study on growth cone collapse. J Neurobiol 24:37-50.

Caroni P, Schwab ME (1988) Two membrane protein fractions from rat central myelin with inhibitory properties for neurite growth and fibroblast spreading. J Cell Biol 106:1281-1288.

Cohen J, Burne JF, McKinlay C, Winter J (1987) The role of laminin and the laminin-fibronectin receptor complex in the outgrowth of retinal ganglion cell axons. Dev Biol 122:407-418.

Drescher U, Kremoser C, Handwerker C, Löschinger J, Noda M, Bonhoeffer $F$ (1995) In vitro guidance of retinal ganglion cell axons by RAGS, a $25 \mathrm{kDa}$ tectal protein related to ligands for eph-receptor tyrosine kinases. Cell 82:359-370.

Godement P, Bonhoeffer F (1989) Cross-species recognition of tectal cues by retinal fibers in vitro. Development 106:313-330.

Hankin MH, Lund RD (1991) How do retinal axons find their targets in the developing brain? Trends Neurosci 14:224-228.

Jhaveri S, Edwards MA, Schneider GE (1991) Initial stages of retinofugal axon development in the hamster: evidence for two distinct modes of growth. Exp Brain Res 87:371-382.

Kennedy TE, Tessier-Lavigne M (1995) Guidance and induction of branch formation in developing axons by target-derived diffusible factors. Curr Opin Neurobiol 5:83-90.

Roskies AL, O'Leary DDM (1994) Control of topographic retinal axon branching by inhibitory membrane-bound molecules. Science 265:799-803.
Simon DK, O’Leary DDM (1992a) Development of topographic order in the mammalian retinocollicular projection. J Neurosci 12:1212-1232.

Simon DK, O'Leary DDM (1992b) Responses of retinal axons in vivo and in vitro to position-encoding molecules in the embryonic superior colliculus. Neuron 9:977-989.

Simon DK, Roskies AL, O'Leary DDM (1994) Plasticity in the development of topographic order in the mammalian retinocollicular projection. Dev Biol 162:384-393.

Stahl B, Müller B, von Boxberg Y, Cox EC, Bonhoeffer F (1990) Biochemical characterization of a putative axonal guidance molecule of the chick visual system. Neuron 5:735-743.

Vielmetter J, Stuermer CAO (1989) Goldfish retinal axons respond to position-specific properties of tectal cell membranes in vitro. Neuron 2:1331-1339.

Vielmetter J, Walter J, Stuermer CAO (1991) Regenerating retinal axons of goldfish respond to a repellent guiding component on caudal tectal membranes of adult fish and embryonic chick. J Comp Neurol 311:321-329.

von Boxberg Y, Deiss S, Schwarz U (1993) Guidance and topographic stabilization of nasal chick retinal axons on target-derived components in vitro. Neuron 10:345-357.

Walter J, Henke-Fahle S, Bonhoeffer F (1987a) Avoidance of posterior tectal membranes by temporal axons. Development 101:909-913.

Walter J, Kern-Veits B, Huf J, Stolze B, Bonhoeffer F (1987b) Recognition of position-specific properties of tectal cell membranes by retinal axons in vitro. Development 101:685-696.

Wizenmann A, Thies E, Klostermann S, Bonhoeffer F, Bähr M (1993) Appearance of target-specific guidance information for regenerating axons after CNS lesions. Neuron 11:975-983. 